\title{
NÚMERO DE CASOS DE SÍFILIS CONGÊNITA NO BRASIL ENTRE OS ANOS DE 2009 A 2013
}

\section{ARTIGO ORIGINAL}

ROCHA, Karina Dias ${ }^{1}$

FECURY, Amanda Alves ${ }^{2}$

OLIVEIRA, Euzébio ${ }^{3}$

DENDASCK, Carla Viana ${ }^{4}$

DIAS, Cláudio Alberto Gellis de Mattos ${ }^{5}$

ROCHA, Karina Dias. Et al. Número de casos de sífilis congênita no Brasil entre os anos de 2009 a 2013. Revista Científica Multidisciplinar Núcleo do Conhecimento. Ano 05, Ed. 05, Vol. 01, pp. 131-143. Maio de 2020. ISSN: 2448-0959, Link de acesso: https://www.nucleodoconhecimento.com.br/saude/casos-de-sifilis, DOI: 10.32749/nucleodoconhecimento.com.br/saude/casos-de-sifilis

1 Técnico em mineração pelo Instituto de Ensino Básico, Técnico e tecnológico do Amapá (IFAP).

${ }^{2}$ Biomédica, Doutora em Doenças Topicais, Professora e pesquisadora do Curso de Medicina do Campus Macapá, Universidade Federal do Amapá (UNIFAP).

3 Biólogo, Doutor em Doenças Topicais, Professor e pesquisador do Curso de Educação Física da, Universidade Federal do Pará (UFPA).

${ }^{4}$ Teóloga, Doutora em Psicanálise, pesquisadora do Centro de Pesquisa e Estudos Avançados- CEPA.

${ }^{5}$ Biólogo, Doutor em Teoria e Pesquisa do Comportamento, Professor e pesquisador do Curso de Licenciatura em Química do Instituto de Ensino Básico, Técnico e tecnológico do Amapá (IFAP). 


\section{RESUMO}

A sífilis é uma doença causada pela bactéria Treponema pallidum. A sífilis congênita é uma doença contagiosa transmitida da mãe para o feto. O objetivo deste trabalho é mostrar o número de casos de sífilis congênita no Brasil entre os anos de 2009 a 2013. A pesquisa foi realizada no banco de dados DATASUS[7]. O número de casos de sífilis congênita aumentou entre os anos de 2009 e 2013 e a região sudeste apresentou a maior média nacional neste período. gênero feminino apresentou um maior número de casos do que o gênero masculino e a maior parte dos casos foram diagnosticados na faixa etária de até seis dias de vida. Uma média três vezes maior de mulheres realizaram pré-natal do que as que não realizaram. Mulheres com grau escolar materno da $5^{\underline{a}}$ a $8^{\underline{a}}$ série incompleta do ensino fundamental apresentaram maior número de casos de sífilis. Percebe-se um maior número de tratamentos da mãe no ano de 2012. No Brasil, apesar da elevada realização de pré-natal, maior parte dos casos de sífilis congênita foi diagnosticado somente após o parto, evidenciando erros no diagnóstico da doença durante a realização do exame e resultando em tratamentos tardios. A escolaridade e a renda são fatores que parecem influenciar no diagnóstico e tratamento da doença. Parece ser necessário que se aumente o número de tratamento dos parceiros, reduzindo assim a transmissão de sífilis e consequentemente a de sífilis congênita. $\mathrm{O}$ tratamento realizado em maior parte dos casos parece garantir a cura da doença.

Palavras-Chave: Sífilis congênita, epidemiologia, Brasil.

\section{INTRODUÇÃO}

A sífilis é uma doença causada pela bactéria Treponema pallidum (SONDA et al., 2013). Ela pode ser transmitida principalmente por contato sexual. Pode ocorrer ainda contaminação por transfusão sanguínea, contato com o mucocutâneo e através da placenta (SANTOS e ANJOS, 2009).

Os sintomas da sífilis dependem do estágio da doença, que é classificada em primária, secundária e terciaria. No primeiro estágio, os sintomas são as lesões no local da 
contaminação, sem manifestações clínicas nas extremidades. No segundo estágio, os sintomas podem ser cefaleia, mal-estar, dor nos ossos, erupção na face e corpo, podendo o sintoma do primeiro estágio ainda estar presente. No terceiro estágio, os sintomas podem ser neurosífilis, psicose, acidente vascular cerebral e perda parcial de movimentos, podendo atingir outros órgãos do corpo (SANTOS e ANJOS, 2009). O tratamento para todos os estágios é realizado com o uso de penicilina e deve se estender aos parceiros sexuais (SONDA et al., 2013).

A sífilis congênita é a doença contagiosa causada pela bactéria Treponema pallidum, quando transmitida da mãe para o feto por via placentária. (PIRES et al., 2014).

O tratamento para sífilis congênita é realizado com o uso de penicilina G cristalina por via endovenosa. Existe uma variação de doses de acordo com as idades, com mães inadequadamente tratadas ou mães tratadas com recém-nascidos que apresentam teste de sífilis negativo (SONDA et al., 2013).

No Brasil, em 2013, haviam 13.705 casos de sífilis congênita, sendo, 4.417 na região nordeste, 1064 na região norte, 5.907 na região sudeste, 1.566 na região sul e 751 na região centro - oeste. No mesmo ano no estado do Amapá contabilizava 98 casos de sífilis congênita (BRASIL, 2016).

\section{OBJETIVO}

Mostrar o número de casos de sífilis congênita no Brasil entre os anos de 2009 a 2013.

\section{MÉTODO}

A pesquisa foi realizada no banco de dados DATASUS[8]. Foram coletados dados nacionais de acordo com as seguintes etapas: A) acessou-se o link[9], clicou-se em "epidemiológicas e Morbidade". Na página aberta foi-se a "Doenças e Agravos de notificação- de 2007 em diante (SINAN)". Na próxima página selecionou-se "Sífilis congênita". Na caixa "abrangência geográfica" optou-se por "Brasil por Região, UF e município". A partir daí seguiram-se os passos: A) Na linha, selecionou-se "Ano Diagnostico", na coluna "não ativa" e no conteúdo "casos confirmados". Todos os 
dados, neste e nos próximos passos, foram coletados entre os anos de 2009 e 2013. B) Na linha, selecionou-se "Região de Notificação", na coluna "Não Ativa" e no conteúdo "casos confirmados"; C) na linha, selecionou-se "Faixa Etária", na coluna "não ativa" e no conteúdo "casos confirmados"; D) Na linha, selecionar "Realizou Prénatal", na coluna selecionar "não ativa", no conteúdo selecionar "casos confirmados"; E) Na linha selecionar "Sífilis Materna", na coluna selecionar "não ativa", no conteúdo, selecionar "casos confirmados"; F) Na linha, selecionar "Ano Diagnóstico", na coluna, selecionar "sexo", no conteúdo, selecionar "casos confirmados". A compilação dos dados foi feita dentro do aplicativo Excel, componente do pacote Office da Microsoft Corporation. A pesquisa bibliográfica foi realizada em artigos científicos, utilizando-se para busca computadores do laboratório de informática do Instituto Federal de Educação, Ciência e Tecnologia do Amapá, Campus Macapá, situado na: Rodovia BR 210 KM 3, s/n - Bairro Brasil Novo. CEP: 68.909-398, Macapá, Amapá, Brasil.

\section{RESULTADOS}

A figura 1 mostra a porcentagem de casos confirmados de sífilis congênita no Brasil entre os anos de 2009 a 2012. No gráfico, nota-se um aumento entre os anos de 2009 e 2012, ocorrendo uma diminuição significativa no ano de 2013.

Figura 1 Mostra a porcentagem de casos confirmados de sífilis congênita no Brasil entre 2009 e 2013.

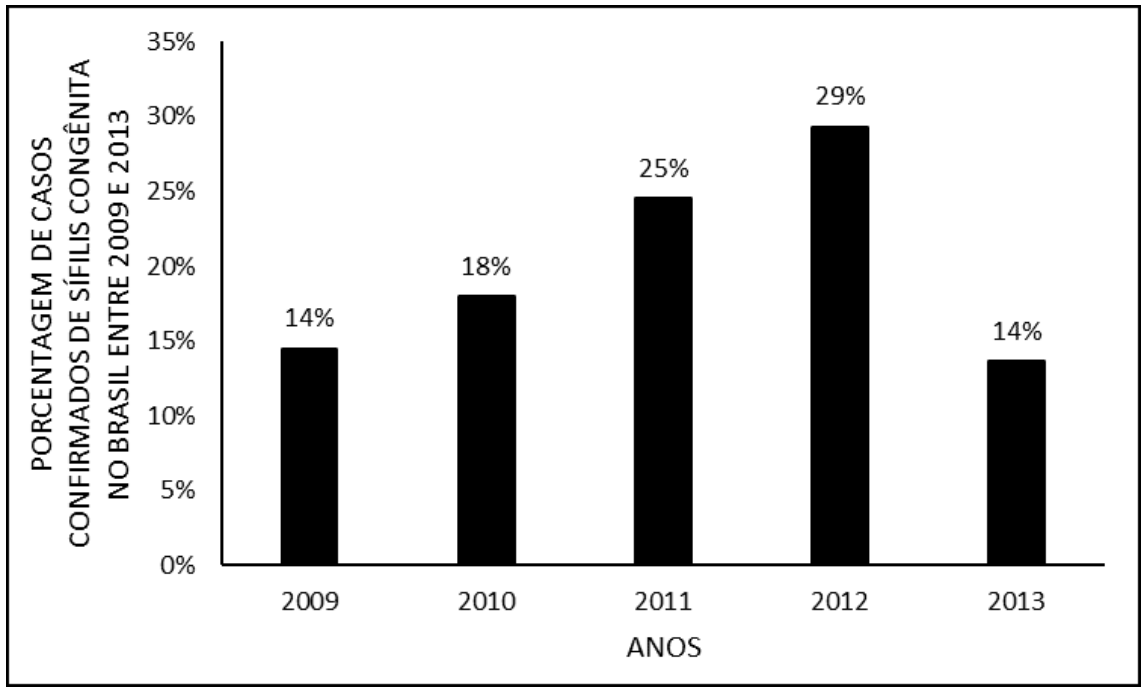


A figura 2 mostra a porcentagem de casos de sífilis congênita por regiões do Brasil entre os anos de 2009 e 2013. A região sudeste apresentou a maior número nacional neste período, seguida da região nordeste. Os menores resultados foram das regiões sul, norte e centro-oeste, respectivamente.

Figura 2 Mostra a porcentagem de casos de sífilis congênita por regiões do Brasil entre 2009 e 2013.

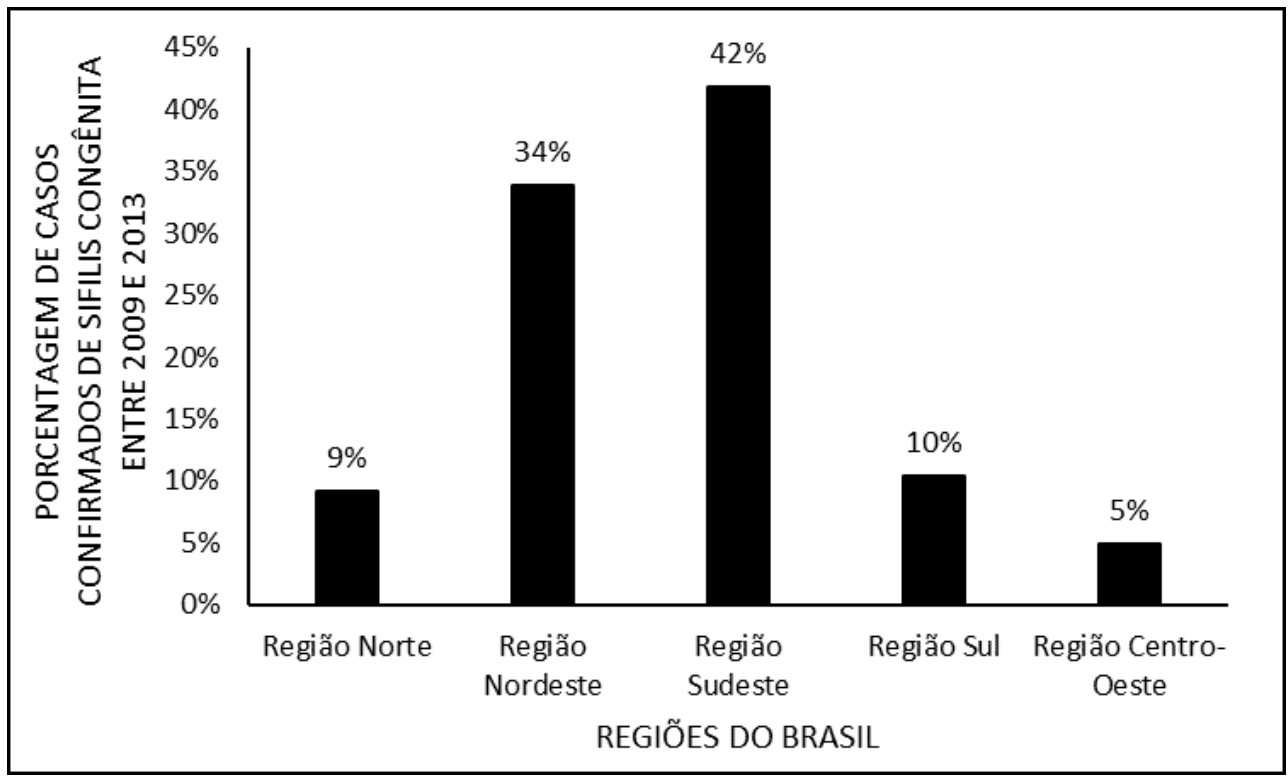

A figura 3 mostra a porcentagem de casos de sífilis congênita por gênero no Brasil, entre os anos de 2009 e 2013. Os dados mostram uma quantidade discretamente maior de casos entre os indivíduos do gênero feminino do que do gênero masculino. 
Figura 3 Mostra a porcentagem de casos de sífilis congênita por gênero no Brasil entre 2009 e 2013.

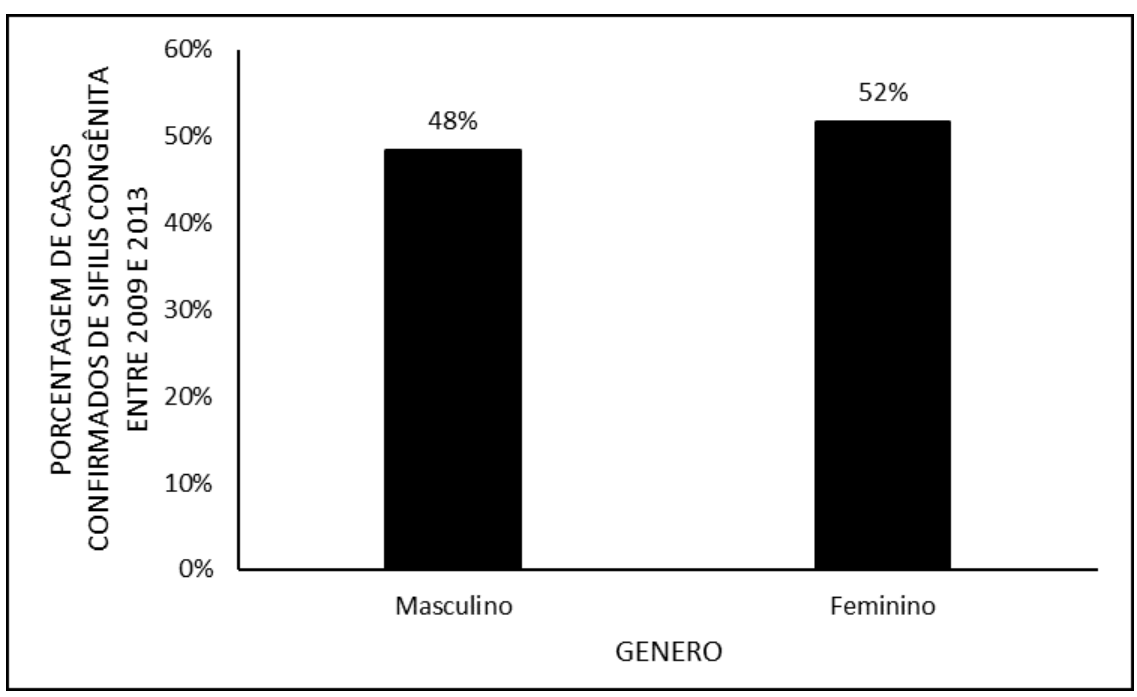

A figura 4 mostra a porcentagem de casos confirmados de sífilis congênita por faixa etária no Brasil entre os anos de 2009 e 2013. No gráfico, percebe-se uma maior quantidade de casos na faixa etária de até seis dias de vida.

Figura 4 Mostra a porcentagem de casos de sífilis congênita por faixa etária no Brasil entre 2009 e 2013.

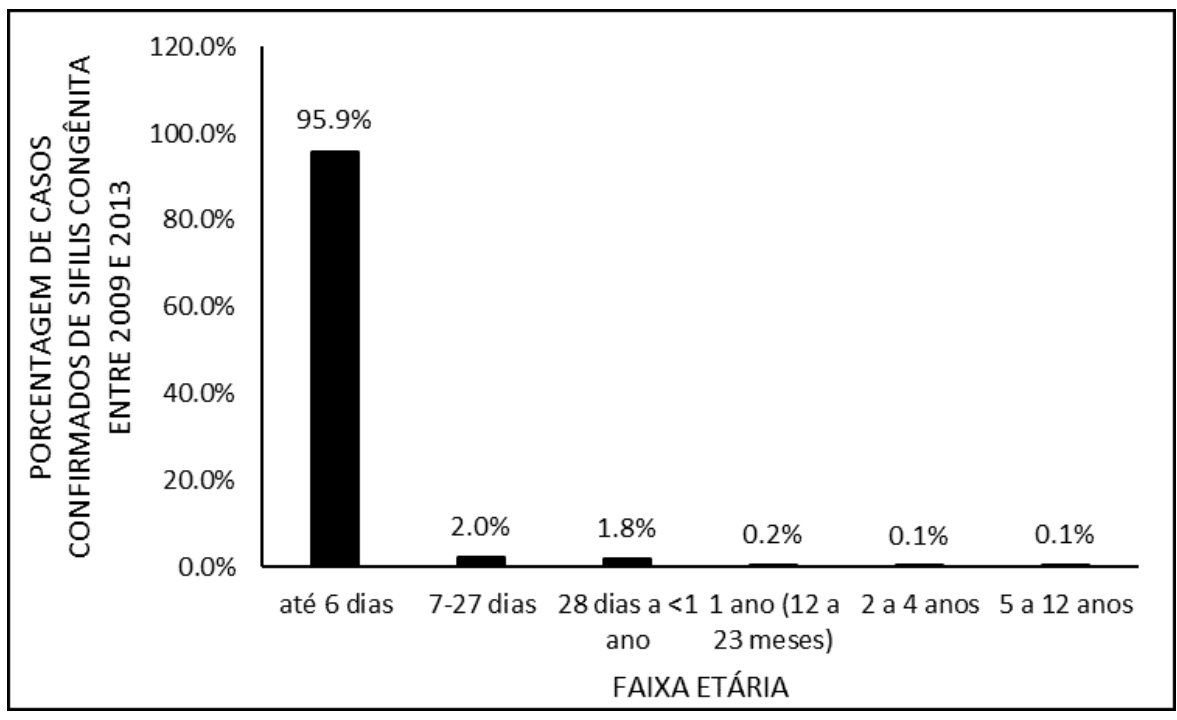


A figura 5 mostra a porcentagem de casos confirmados de sífilis congênita no Brasil por realização de exame pré natal entre os anos de 2009 e 2013. Os dados mostram uma porcentagem maior de mulheres que realizaram pré-natal do que as que não realizaram.

Figura 5 Mostra a porcentagem de casos confirmados de sífilis congênita no Brasil por realização de exame pré natal entre 2009 e 2013.

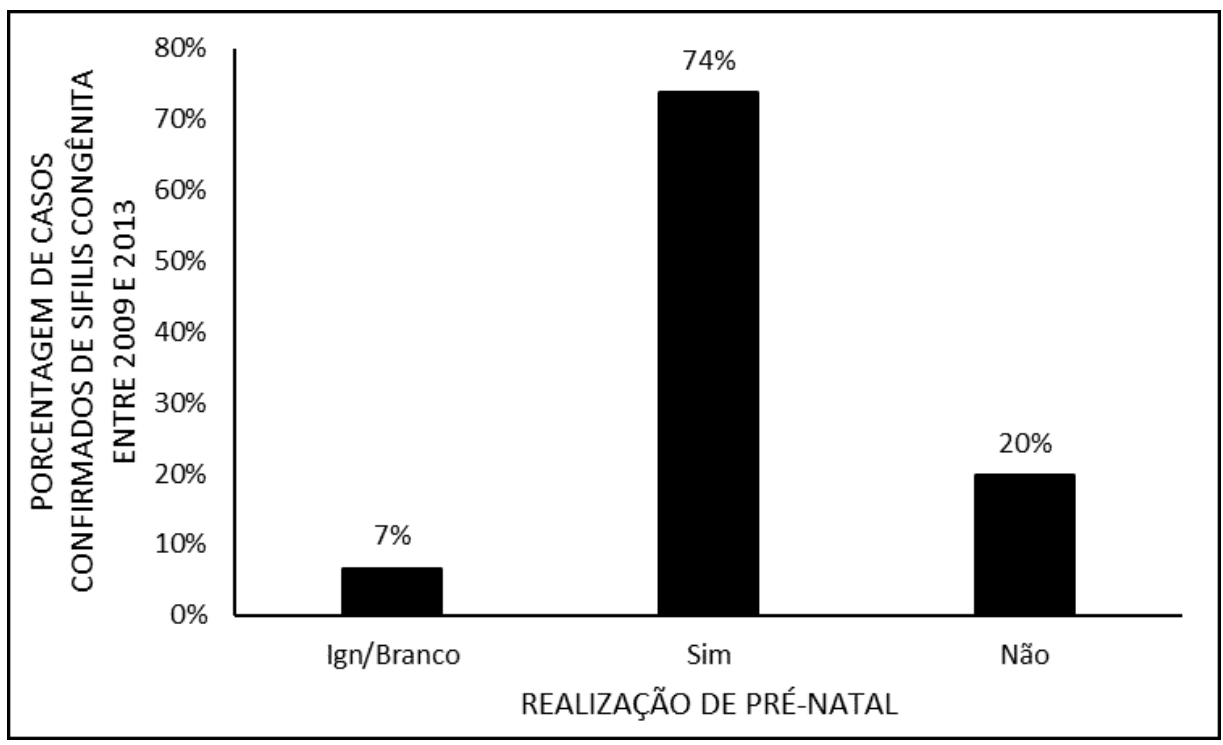

A figura 6 mostra a porcentagem de casos confirmados de sífilis congênita no Brasil pelo momento de infecção materna por sífilis, entre os anos de 2009 e 2013. Percebese uma maior quantidade de infecções durante o exame pré-natal e também momento do parto. 
Figura 6 Mostra a porcentagem de casos confirmados de sífilis congênita no Brasil pelo momento de infecção materna por sífilis, entre os anos de 2009 e 2013

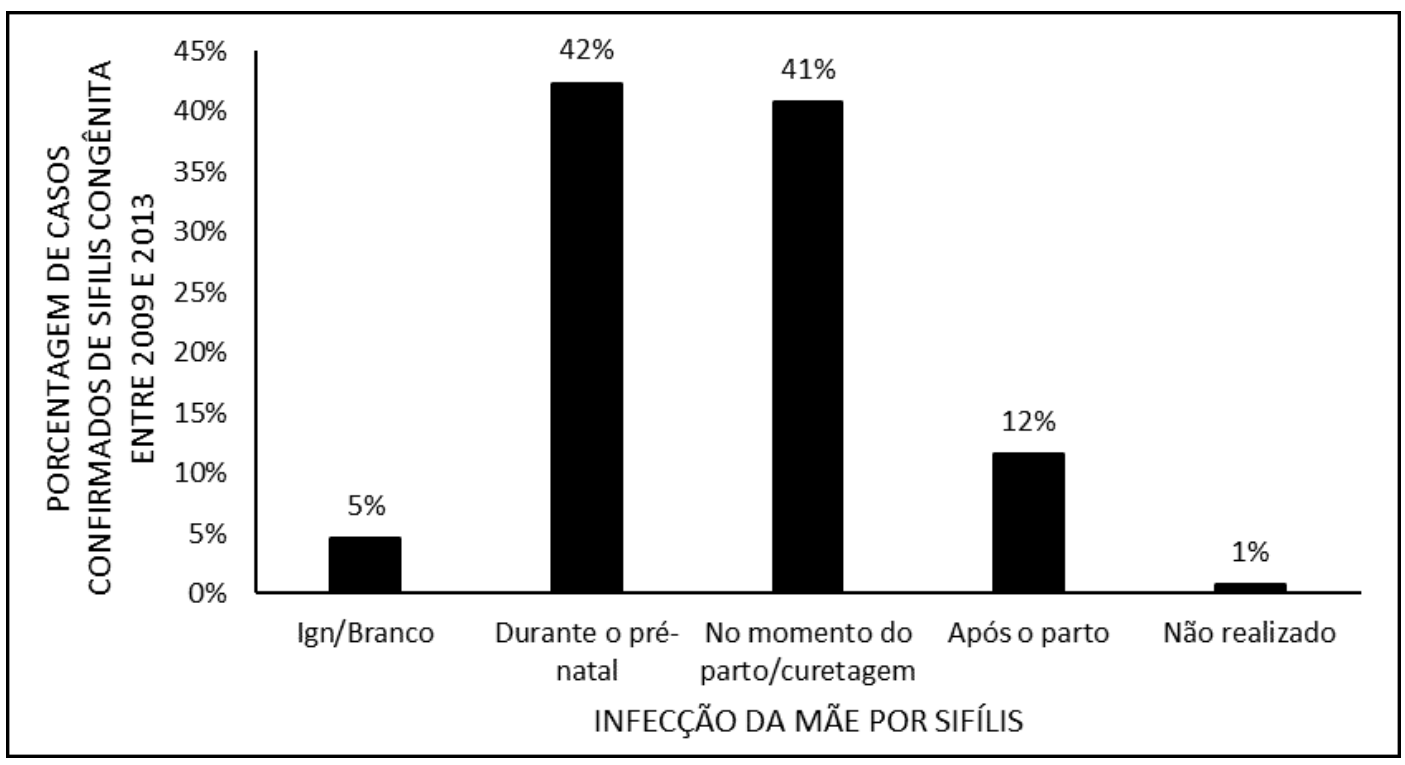

A figura 7 mostra a porcentagem de casos confirmados de sífilis congênita no Brasil pelo grau de escolaridade da mãe entre os anos de 2009 e 2013. Os dados mostram uma maior quantidade de casos com o grau escolar materno da $5^{\underline{a}}$ a $8^{\underline{a}}$ série incompleta do ensino fundamental, e o menor número com a educação superior completa.

Figura 7 Mostra a porcentagem de casos confirmados de sífilis congênita no Brasil pelo grau de escolaridade da mãe entre os anos de 2009 e 2013

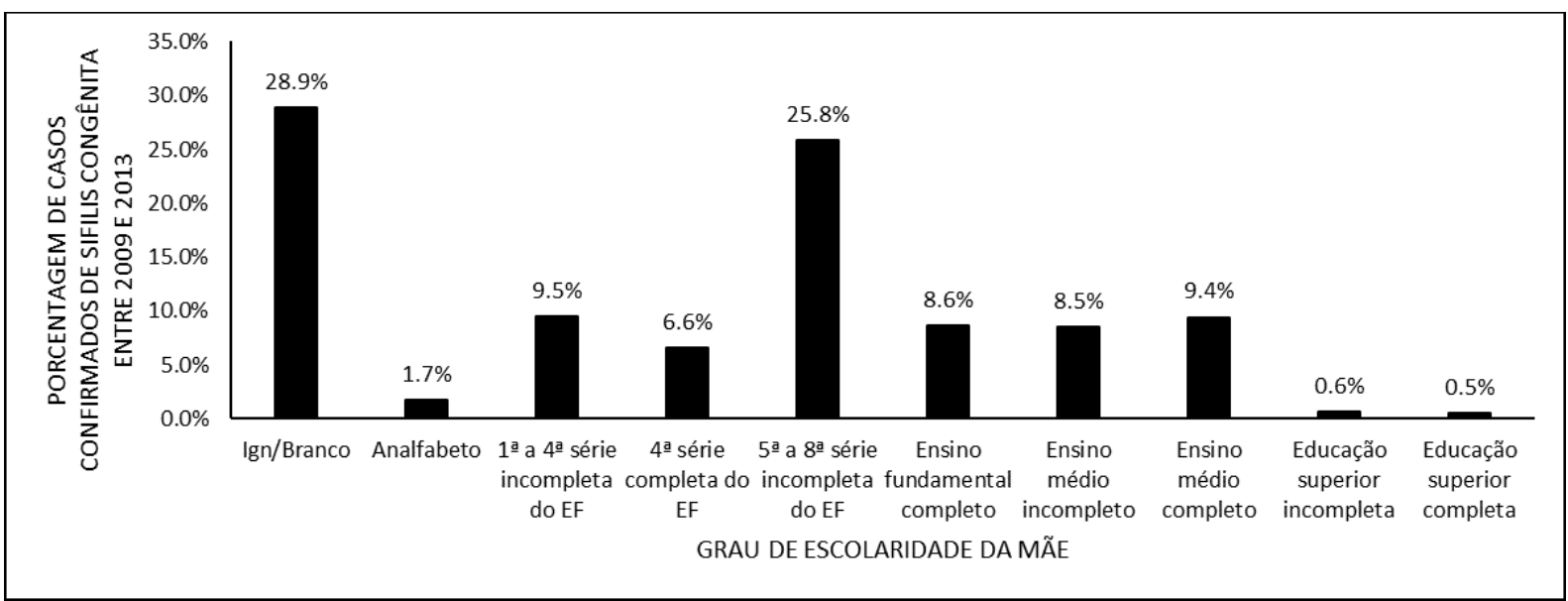


A figura 8 mostra a porcentagem de casos confirmados de sífilis congênita no Brasil por etnia (raça) entre os anos de 2009 e 2013. A etnia parda apresentou a maior quantidade de casos, seguida pela branca. As etnias que apresentaram menor número foram preta, indígena e amarela, respectivamente.

Figura 8 Mostra a porcentagem de casos confirmados de sífilis congênita no Brasil por etnia (raça) entre os anos de 2009 e 2013.

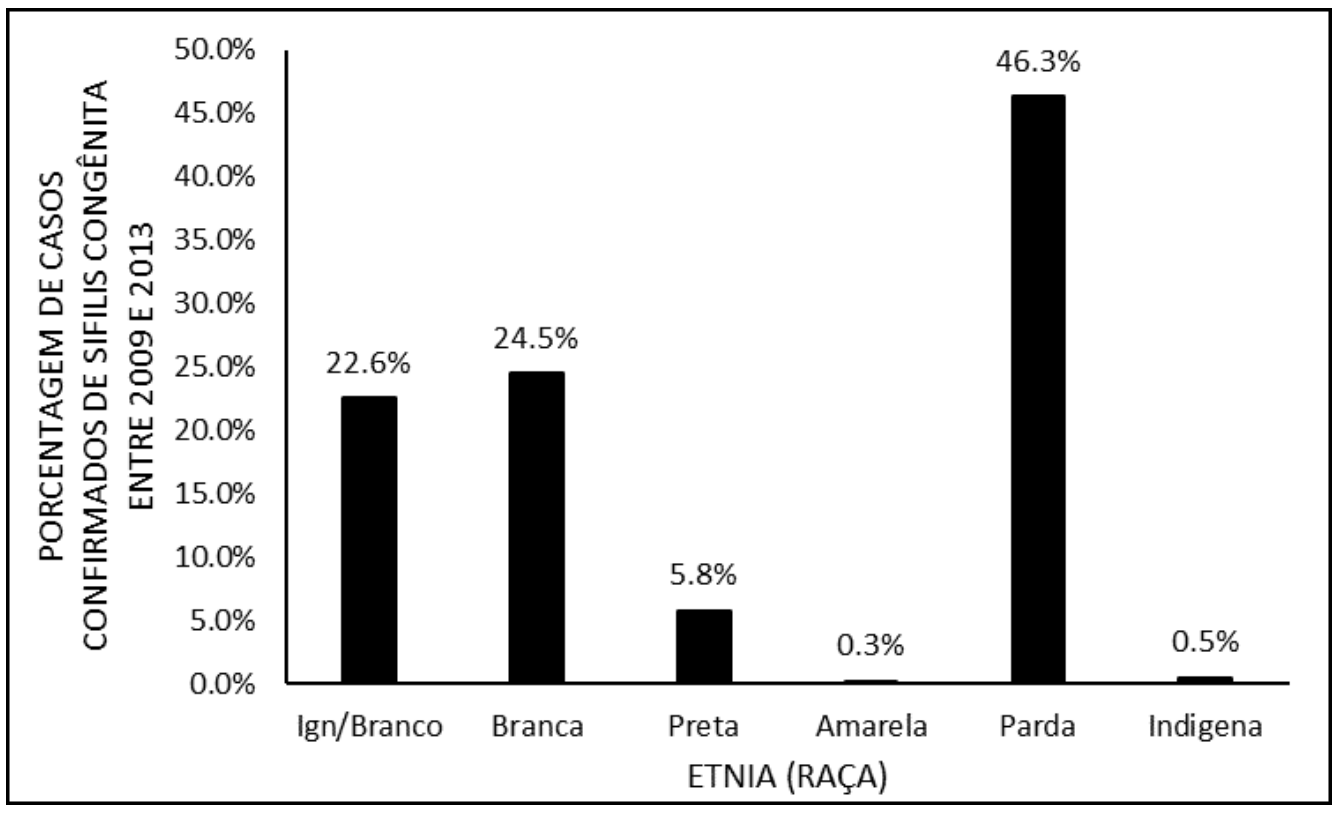

A figura 9 mostra a porcentagem de casos confirmados de sífilis congênita no Brasil pelo ano de início de tratamento da mãe entre os anos de 2009 e 2013. Percebe-se um maior número de tratamentos da mãe no ano de 2012, seguido pelo ano de 2011. O ano de 2013 apresentou o menor número, seguido pelo ano de 2009 e 2010. 
Figura 9 Mostra a porcentagem de casos confirmados de sífilis congênita no Brasil pelo ano de início de tratamento da mãe entre os anos de 2009 e 2013.

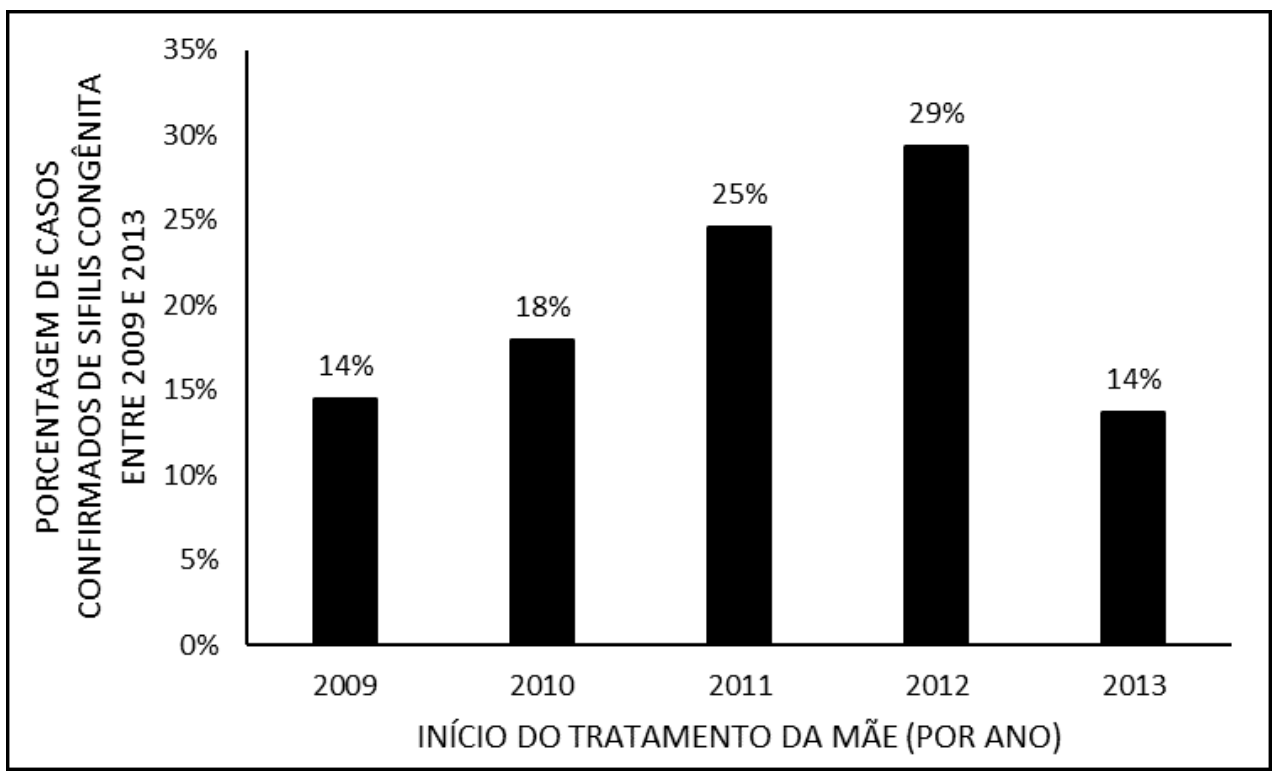

A figura 10 mostra a porcentagem de casos confirmados de sífilis congênita por tratamento do parceiro entre os anos e 2009 e 2013. Os dados mostram um número maior de homens que não realizaram o tratamento do que os que não realizaram.

Figura 10 Mostra a porcentagem de casos confirmados de sífilis congênita por tratamento do parceiro entre os anos e 2009 e 2013.

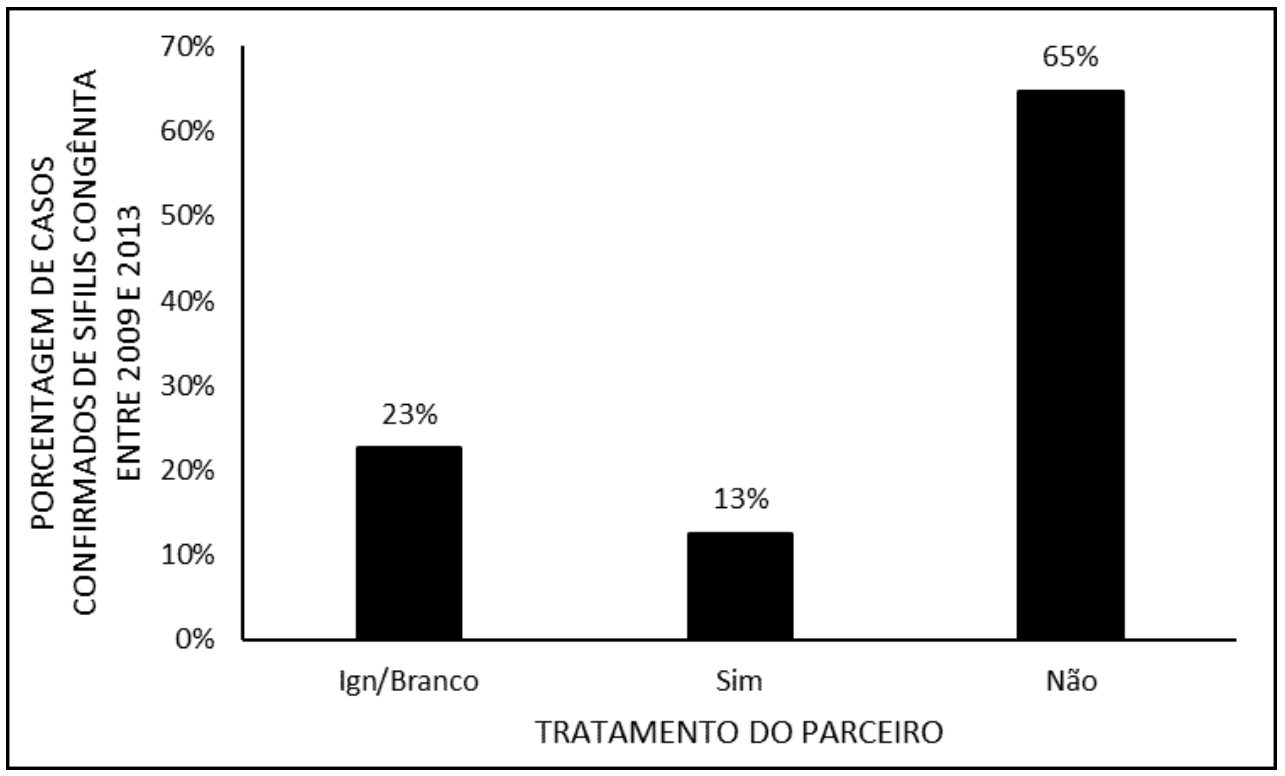


A figura 11 mostra porcentagem de casos confirmados de sífilis congênita no Brasil por evolução entre 2009 e 2013. No gráfico, nota-se um número expressivamente maior de vivos do que de óbitos pelo agravo notificado ou por outra causa.

Figura 11 Mostra porcentagem de casos confirmados de sífilis congênita no Brasil por evolução entre 2009 e 2013.

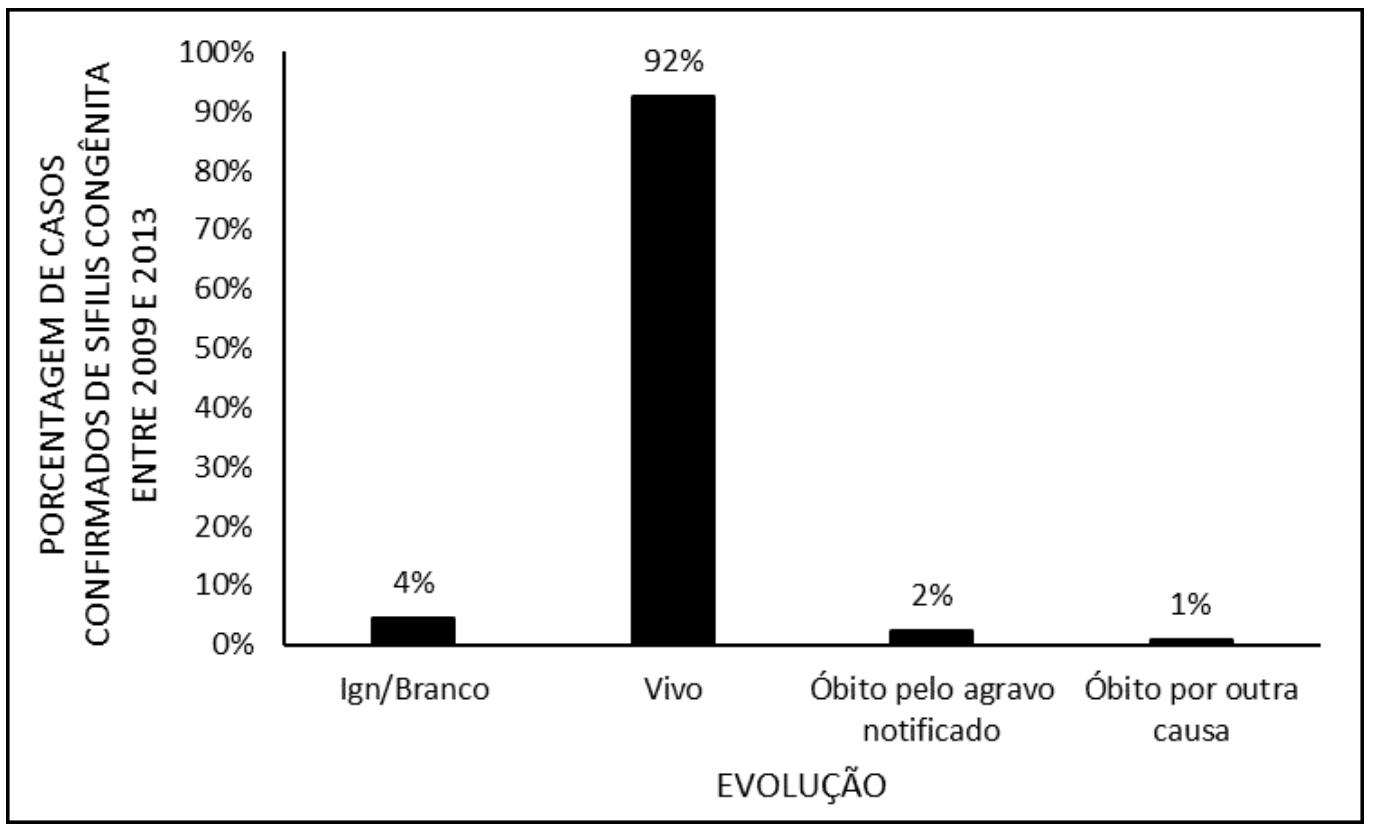

\section{DISCUSSÃO}

Os dados mostram um aumento entre os anos de 2009 e 2013, ocorrendo uma diminuição significativa no ano de 2013 (figura 1). Provavelmente, a causa foi a campanha lançada pelo Ministério Público em 2013, que incentivou o diagnóstico da doença durante o pré-natal, além de aumentar a oferta de testes para o diagnóstico da sífilis de 31,5 mil em 2011 para cerca de 1,7 milhão em 2013. O aumento do número de casos confirmados de sífilis congênita entre 2009 e 2012, pode ser explicado pelo crescimento do número de unidades de saúde notificadoras, possibilitando o acesso a exames e teste, e permitindo que vários casos entrassem para os registros (BRASIL, 2014; 2017). 
Entre 2009 e 2013, a região sudeste do Brasil apresentou o maior número de casos de sífilis congênita (figura 2). Provavelmente, a causa é o grande contingente populacional da região que, segundo o IBGE, tem cerca de 80 milhões de habitantes, sendo a região mais populosa do país. Isso pode ocasionar um maior número de relações sexuais sem proteção, além de aumentar o risco de transmissão por tecido mucocutâneo e transfusão sanguínea, acometendo assim as grávidas que transmitem a doença para o feto (GUIMARÃES et al., 2015).

Entre 2009 e 2013, cerca de 52\% dos casos de sífilis congênita foram diagnosticados no gênero feminino (figura 3). O fato de não ter um parceiro sexual fixo, associado ao não uso de preservativo, justificado em maior parte dos casos pela confiança nos parceiros sexuais, explicam a doença acometer mais as mulheres (MENESES et al., 2017; MOREIRA et al., 2017).

Entre 2009 e 2013 o maior número de casos de sífilis congênita foi diagnosticado na faixa etária de até 6 dias de vida (figura 4). Parece ocorrer uma não realização do prénatal ou a falha no diagnóstico da doença durante o exame que faz com que a sífilis congênita seja diagnosticada somente após o parto. A criança que apresenta sintomas logo após o nascimento força a realização de exames e testes para diagnosticar a doença (DOMINGUES et al., 2013).

Entre os anos de 2009 e 2013, 74\% das gestantes realizaram o pré-natal (figura 5). Provavelmente a causa é o incentivo do governo federal à assistência básica de saúde, elevando assim a cobertura da assistência pré-natal. Porém, nota-se que essa assistência foi de baixa qualidade resultando em erros no diagnóstico de sífilis congênita no momento do exame (PEDRAZA, 2016).

Uma maior porcentagem de casos confirmados de sífilis congênita por sífilis materna no Brasil parece ser detectada no exame prenatal e momento do parto (figura 6). A desigualdade econômica no Brasil faz com que um grupo majoritário de gestantes realize um exame pré-natal precário que pode resultar em erros no diagnóstico e não detectar a doença. Devido a isto os casos de sífilis congênita foram diagnosticados 
durante o exame e a maior parte dos casos foram detectados no momento do parto (SANTOS et al., 2016).

No período da pesquisa os dados mostram um maior número de casos em pessoas com escolaridade mais baixa (figura 7). Provavelmente gestantes com baixa escolaridade tendem a não realizar o pré-natal, por não ter acesso, desconhecer, ou desvalorizar a sua importância. Este fato parece impossibilitar o tratamento durante a gravidez (SANTOS et al., 2016).

No Brasil, a autodeclaração de raça é garantida por lei (CRUZ, 2016). Entre os anos de 2009 e 2013, a etnia parda apresentou o maior número de casos registrados (figura 8). Provavelmente a mistura de raças decorrente da colonização do país resultou em uma população onde grande parte das pessoas se declaram pardas (MOREIRA et al., 2017).

Entre 2009 e 2012, houve um aumento do número de tratamentos da mãe, ocorrendo uma queda significativa no ano de 2013. A redução do número de casos confirmados de sífilis congênita pode ter acontecido em decorrência da campanha realizada pelo Ministério Público que incentivou o diagnóstico da doença durante a gravidez (BRASIL, 2017).

No país, entre 2009 e 2013, 65\% dos parceiros sexuais de mulheres diagnosticadas com sífilis durante a gravidez não realizaram o tratamento contra doença (figura 10). Os motivos provavelmente são a falta de conhecimento sobre a doença e de suas consequências, além da associação cultural do diagnóstico de sífilis à infidelidade (FIGUEIREDO et al., 2015).

A pesquisa mostra uma porcentagem grande de vivos em relação aos óbitos pelo agravo notificado e por outros. Entre 2009 e 2013, cerca de 92\% dos casos de sífilis congênita sobreviveram à doença e $2 \%$ foram a óbito. Possivelmente o tratamento realizado nos casos registrados parece garantir a cura do agravo (MOREIRA et al., 2017). 


\section{CONCLUSÃO}

No Brasil, apesar da elevada realização de pré-natal, maior parte dos casos de sífilis congênita foi diagnosticado somente após o parto, evidenciando erros no diagnóstico da doença durante a realização do exame e resultando em tratamentos tardios. A escolaridade e a renda são fatores que parecem influenciar no diagnóstico e tratamento da doença. Parece ser necessário que se aumente o número de tratamento dos parceiros, reduzindo assim a transmissão de sífilis e consequentemente a de sífilis congênita. $O$ tratamento realizado em maior parte dos casos parece garantir a cura da doença.

\section{REFERÊNCIAS}

BRASIL. Boletim Epidemiológico de DST/Aids. Brasília DF: Ministério da Saúde, 2014. 84p.

. Sífilis 2016. Brasilia DF: Ministério da Saúde: 29 p. 2016.

- Ministério da Saúde Lança Campanha de Combate à Sífilis. Brasilia DF, 2017. Disponível em: < http://www.brasil.gov.br/saude/2013/10/ministerio-dasaude-lanca-campanha-de-combate-a-sifilis >. Acesso em: 26 set 2017.

CRUZ, I. C. F. Notas sobre prevenção da fraude - autodeclaração sobre raça/cor. Boletim NEPAE-NESEN, v. 13, n. 1, p. 1-4, 2016.

DOMINGUES, R. M. S. M. et al. Sífilis congênita: evento Sentinela da qualidade da Assistência pré-natal. Revista Saúde Pública, v. 47, n. 1, p. 147-157, 2013.

FIGUEIREDO, M. S. N. et al. Percepção de enfermeiros sobre a adesão ao tratamento dos parceiros de gestantes com sífilis. Revista Rene, v. 16, n. 3, p. 345354, 2015. 
GUIMARÃES, R. M. et al. Diferenças regionais na transição da mortalidade por doenças cardiovasculares no Brasil, 1980 a 2012. Revista Panamericana de Salud Pública, v. 37, n. 2, p. 83-89, 2015.

MENESES, M. O. et al. O Perfil do Comportamento Sexual de Risco de Mulheres Soropositivas para Sífilis. Revista de Enfermagem, v. 11, n. 4, p. 1584-1594, 2017.

MOREIRA, K. F. A. et al. Perfil dos Casos Notificados de Sífilis Congênita Cogitare Enfermagem, v. 22, n. 2, p. 1-10, 2017.

PEDRAZA, D. F. Assistência ao pré-natal, parto e pós-parto no município de Campina grande, Paraíba. Cadernos Saúde Coletiva v. 24, n. 4, p. 460-467, 2016.

PIRES, A. C. S. et al. Ocorrência de sífilis congênita e os principais fatores relacionados ao índice transmissão da doença no Brasil da atualidade. Revista Uningá, v. 19, n. 1, p. 58-64, 2014.

SANTOS, G. S. et al. Aspectos Epidemiológicos da sífilis Congênita Associados à Escolaridade Materna. Revista de Enfermagem, v. 10, n. 8, p. 2845-2852, 2016.

SANTOS, V. C.; ANJOS, K. F. Sífilis: Uma realidade prevenível. Sua erradicação, um desafio atual. Revista Saúde e Pesquisa, v. 2, n. 2, p. 257-263, 2009.

SONDA, E. C. et al. Sífilis Congênita: uma revisão da literatura. Revista de Epidemiologia e controle de Infecção, v. 3, n. 1, p. 28-30, 2013.

\section{APÊNDICE - REFERÊNCIAS DE NOTA DE RODAPÉ}

7. http://datasus.saude.gov.br/

8. http://datasus.saude.gov.br/

9. datasus.gov.br

Enviado: Maio, 2020. 
Aprovado: Maio, 2020. 